\title{
Biosurveillance Adaptable Framework for Teaming, Exploration and Reuse (BioAFTER)
}

\author{
Timothy Dasey* and Lars Fiedler \\ Chemical and Biological Defense Systems, MIT Lincoln Laboratory, Lexington, MA, USA
}

\section{Introduction}

Next-generation software environments for disease surveillance will need to have several important characteristics, among which are collaboration and search and discovery features, access to various data sets, and a variety of analytic methods. However, perhaps the most important feature is the least often mentioned - the ability to have the system adapt over time without high reengineering cost. The public health community cannot afford software redesigns every few years as data sets expand, analysis needs evolve, and software deficiencies are exposed. In addition to the need to adapt an environment over longer time periods, epidemiologists have high variability in their day-to-day needs that require adaptability over short time periods as well. Each outbreak or health situation has unique aspects, and analysts need to be able to bring in data and methods unique to that situation that may not be easily anticipated a priori.

The most common approach to increasing reusability and decreasing upgrade costs are open architecture software frameworks such as Service-Oriented Architectures (SOAs). If well implemented, SOAs can significantly reduce software upgrade costs by allowing services (a software module) to be easily swapped out for improvements or supplemented with additional services. SOAs can help with long-term adaptability, but are not useful in short-term adaptability, since the software development team must be engaged in each cycle. Another approach is to include an App Store. Unfortunately, App Stores for government use have often been disappointing. Apps can tend to be quite simple, and even slight changes from what is programmed - a predictable situation with the variability seen in disease surveillance realm - will result in an epidemiologist having to get a software developer to make them a new App.

\section{Methods}

Instead of the power for adaptability remaining solely in the control of software developers, that power needs to also be in the hands of the users themselves. The BioAFTER project builds upon SOA and App Store concepts by allowing Apps to be strung together in unique combinations, according to the problem of the day. As examples, these Apps can be data access programs, data quality editing, algorithms of various complexity, or reporting and visualization modules. The App Store feature allows software developers, including the public health academic community, to add new methods to the environment, while the composability feature allows ad-hoc combinations of Apps to suit particular situations.
The user composability feature would be of limited value without collaboration features in the environment. We expect that only a subset of users will make the effort to do composition. The rest will want to learn from those "super users". The BioAFTER environment allows data, Apps (including new Apps created by compositions of other Apps), and analysis results to be shared with the entire epidemiological community, or with a set of "friends" or people with common interests. Analysts who use various Apps can rate their value. The environment is intended to allow search and discovery of Apps, expertise, or even people who may have needed expertise or experience with similar situations (these features are in development).

\section{Results}

The motivations and features of the BioAFTER environment will be described at the ISDS Conference, and a brief demonstration of the capabilities on an example use case will be given.

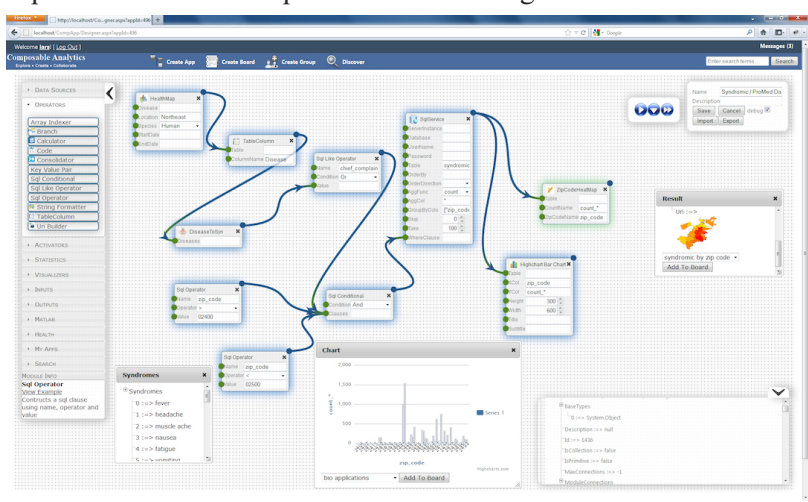

\section{Keywords}

biosurveillance; collaboration; discovery; composition; adaptability

\section{Acknowledgments}

This work is sponsored by the Office of the Assistant Secretary of Defense for Research and Engineering (ASD(R\&E)) under Air Force Contract \#FA8721-05-C-0002. Opinions, interpretations, recommendations and conclusions are those of the authors and are not necessarily endorsed by the United States Government.

\author{
*Timothy Dasey \\ E-mail:TimD@LL.mit.edu
}

